\title{
NONLINEAR EFFECTS IN ELECTROHYDRODYNAMIC SURFACE WAVE PROPAGATION*
}

\author{
BY \\ D. H. MICHAEL \\ University College London
}

The author has recently made a number of mathematical studies of the effects of electrostatic surface stresses on the equilibrium of menisci and on wave motions at the surface of highly conducting fluids. One aspect of such problems which is of interest is that electrostatic boundary conditions can be applied in different ways, and one may contrast the effects on wave motion when electric fields are produced by insulated charged conductors or by conductors with maintained electrical potentials. A discussion of the problem for linearized theory has recently been given by the author [1], and so far as small-amplitude waves are concerned, there is no difference between these two cases. However, it seems inconceivable that such a similarity could extend into wave motions which are not small, and the following work takes the analysis of plane wave motion to a higher order, with a view to showing how the differing electrostatic conditions do produce different effects.

In Sec. 2 a discussion is given for waves of permanent form, which shows that the behavior of the electric field begins to differ in these two cases in the second-order effects, and that the difference is reflected in different phase velocities. The other features of the wave motion, such as the surface elevation, are indistinguishable at this stage of approximation, and it seems that differences in these features only begin to appear in third- or higher-order approximations.

In Sec. 3 a similar analysis is given for unstable standing waves. Similar second-order differences appear in the electric field, but the effect is now simply to change the mean level of the fluid pressure. The growth rate and the surface elevation are indistinguishable in the second-order analysis.

2. Propagation of progressive waves without change of form. We consider the propagation of waves on a conducting fluid of height $a$ with a conducting plate at a distance $b$ above the surface. The undisturbed free surface of the fluid is at $z=0$, the conducting plate at $z=b$, and the fluid is supported on a conducting plate at $z=-a$. In the first place let the upper plate be maintained at a fixed potential $V=V_{0}$ above the fluid for which $V=$ 0 .

We study a plane progressive wave of wave length $2 \pi / k$ travelling without change of form in the horizontal $x$ direction with phase velocity $U$. We shall refer the analysis to the Newtonian frame moving with speed $U$ relative to the boundaries, in which the wave disturbance is steady, and steady-state equations apply.

\footnotetext{
* Received August 12, 1976; revised version received February 14, 1977. The author wishes to acknowledge the support of the National Research Council of Canada during the preparation of this paper.
} 
Let the electrostatic potential in the air space be $\phi$ and the velocity potential in the fluid $\Omega$. The surface elevation $z=\eta(x)$ due to waves will be a periodic function of wavelength $2 \pi / k$, having components of the form

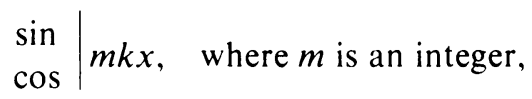

but no constant terms since the mean level of the surface cannot change when the fluid is incompressible.

In terms of a small parameter $\epsilon$ which we shall identify later, we write

$$
\begin{array}{lr}
\phi= & \phi_{0}+\epsilon \phi_{1}+\epsilon^{2} \phi_{2}+\cdots, \\
\eta= & \epsilon \eta_{1}+\epsilon^{2} \eta_{2}+\cdots, \\
\Omega= & \Omega_{0}+\epsilon \Omega_{1}+\epsilon^{2} \Omega_{2}+\cdots,
\end{array}
$$

where $\phi_{0}=V_{0} z / b$, the undisturbed electrostatic potential.

In the chosen frame of reference the fluid has a mean velocity $U$ in the $x$ direction, where

$$
U=U_{0}+\epsilon U_{1}+\epsilon^{2} U_{2}+\cdots
$$

with the notation that the fluid velocity $\mathrm{v}=\operatorname{grad} \Omega, \Omega_{0}=-U_{0} x$.

The solution of the problem to all orders in $\epsilon$ will satisfy the following conditions:

$$
\begin{array}{r}
\int_{0}^{2 \pi / k} \eta d x=0, \\
\nabla^{2} \phi=0, \\
\nabla^{2} \Omega=0
\end{array}
$$

(iv) $\partial \Omega / \partial z=0$ at $z=-a$, implying that $\partial \Omega_{1} / \partial z=\partial \Omega_{2} / \partial z=\partial \Omega_{3} / \partial z=\cdots=0$ at $z=-a$.

(v) For steadiness $\partial \Omega / \partial n$, representing the normal velocity of the fluid, is zero at $z$ $=\eta$.

(vi) The normal stress is continuous at the free surface.

The electrostatic boundary conditions may be of different forms. In (a) and (b) below we consider two cases in turn, the first in which the potentials of the conducting surfaces are kept fixed, and the second when the charge is kept fixed.

(a) Oscillations at fixed potentials. Here the electrostatic condition will be

(vii) $\phi=\phi_{0}$ at $z=b$, which implies that $\phi_{1}=\phi_{2}=\phi_{3}=\cdots=0$ at $z=b$.

(viii) $\phi=0$ at $z=\eta$.

Starting with condition (v), if $\psi$ is the inclination of the surface to the horizontal, $d \eta / d x=\tan \psi$, and the condition is

$$
\frac{\partial \Omega}{\partial x} \frac{d \eta}{d x}-\frac{\partial \Omega}{\partial z}=0
$$

The condition is exact provided we take $\partial \Omega / \partial x$ and $\partial \Omega / \partial z$ at $z=\eta$. To express it correctly in powers of $\epsilon$ we write 


$$
\begin{aligned}
\left\{\left(\frac{\partial \Omega}{\partial x}\right)_{0}+\eta \frac{\partial}{\partial z}\left(\frac{\partial \Omega}{\partial x}\right)_{0}+\frac{\eta^{2}}{2 !} \frac{\partial^{2}}{\partial z^{2}}\left(\frac{\partial \Omega}{\partial x}\right)_{0}\right. & +\cdots\} \frac{\partial \eta}{\partial x} \\
& -\left\{\left(\frac{\partial \Omega}{\partial z}\right)_{0}+\eta\left(\frac{\partial^{2} \Omega}{\partial z^{2}}\right)_{0}+\frac{\eta^{2}}{2 !}\left(\frac{\partial^{3} \Omega}{\partial z^{3}}\right)_{0}+\cdots\right\}=0,
\end{aligned}
$$

in which the suffix 0 denotes that the derivatives of $\Omega$ refer to $z=0$. It is necessary to substitute for $\eta$ from (2) and equate to zero the coefficients of successive powers of $\epsilon$. We find these conditions, up to the terms in $\epsilon^{2}$, to be

$$
\begin{array}{ll}
\epsilon_{1}{ }^{0}: & \frac{\partial \Omega_{0}}{\partial z}=0 \\
\epsilon_{1}{ }^{1}: & \left(\frac{\partial \Omega_{0}}{\partial x}\right) \frac{d \eta_{1}}{d x}-\frac{\partial \Omega_{1}}{\partial z}-\eta_{1} \frac{\partial^{2} \Omega_{0}}{\partial z^{2}}=0, \\
\epsilon_{1}{ }^{2}: & \left(\frac{\partial \Omega_{1}}{\partial x}\right) \frac{d \eta_{1}}{d x}+\left(\frac{\partial \Omega_{0}}{\partial x}\right) \frac{d \eta_{2}}{d x}-\frac{\partial \Omega_{2}}{\partial z}-\eta_{1} \frac{\partial^{2} \Omega_{1}}{\partial z^{2}}=0,
\end{array}
$$

where it is now implied that the derivatives of $\Omega$, and later of $\phi$, refer to $z=0$ without writing in the suffix 0 throughout.

For condition (viii) we have

$$
\phi_{z=\eta}=\phi_{0}+\eta\left(\frac{\partial \phi}{\partial z}\right)_{0}+\frac{\eta^{2}}{2 !}\left(\frac{\partial^{2} \phi}{\partial z^{2}}\right)_{0}+\cdots,
$$

and using (1) and (2) we find the following conditions up to the order $\epsilon^{2}$ :

$$
\begin{array}{ll}
\epsilon^{0}: & \phi_{0}=0, \\
\epsilon^{1}: & \phi_{1}+\eta_{1} \frac{\partial \phi_{0}}{\partial z}=0, \\
\epsilon{ }^{2}: & \phi_{2}+\eta_{2} \frac{\partial \phi_{0}}{\partial z}+\eta_{1} \frac{\partial \phi_{1}}{\partial z}=0 .
\end{array}
$$

In condition (vi) the dynamic pressure $p$ in the fluid is given by Bernoulli's equation

$$
\rho+\frac{1}{2} p(\operatorname{grad} \phi)^{2}=\text { constant }
$$

where $\rho$ is the density. Thus, allowing for surface tension, electrical, and gravitational contributions to the stress we have the condition

$$
\frac{1}{8 \pi}\left(\frac{\partial \phi}{\partial n}\right)^{2}+T \frac{\frac{d^{2} \eta}{d x^{2}}}{\left\{1 \dot{+}\left(\frac{d \eta}{d x}\right)^{2}\right\}^{3 / 2}}-\rho g \eta-\frac{\rho}{2}(\operatorname{grad} \Omega)^{2}=\text { constant }
$$

at $z=\eta$. This condition is to be treated as shown previously. The algebra is lengthy and will be omitted here. The results are as follows, up to terms of order $\epsilon^{2}$ :

$$
\begin{aligned}
& \epsilon_{,}^{0}: \frac{1}{8 \pi}\left(\frac{\partial \phi_{0}}{\partial z}\right)^{2}-\frac{\rho}{2}\left(\frac{\partial \Omega_{0}}{\partial x}\right)^{2}=\text { constant }, \\
& \epsilon_{,}{ }^{1}: \frac{1}{4 \pi} \frac{\partial \phi_{0}}{\partial z} \frac{\partial \phi_{1}}{\partial z}+T \frac{d^{2} \eta_{1}}{d x^{2}}-\rho g \eta_{1}-\rho\left(\frac{\partial \Omega_{0}}{\partial x}\right)\left(\frac{\partial \Omega_{1}}{\partial x}\right)=0,
\end{aligned}
$$




$$
\begin{aligned}
& \epsilon_{.}^{2}: \frac{1}{8 \pi}\left\{\left(\frac{\partial \phi_{1}}{\partial z}\right)^{2}+2 \frac{\partial \phi_{0}}{\partial z}\left(\frac{\partial \phi_{2}}{\partial z}+\eta_{1} \frac{\partial^{2} \phi_{1}}{\partial z^{2}}-\frac{d \eta_{1}}{d x} \frac{\partial \phi_{1}}{\partial x}\right)-\left(\frac{\partial \phi_{0}}{\partial z}\right)^{2}\left(\frac{d \eta_{1}}{d x}\right)^{2}\right\} \\
& +T \frac{d^{2} \eta_{2}}{d x^{2}}-\rho g \eta_{2}-\frac{\rho}{2}\left\{\left(\frac{\partial \Omega_{1}}{\partial x}\right)^{2}+\left(\frac{\partial \Omega_{1}}{\partial z}\right)^{2}+2 \frac{\partial \Omega_{0}}{\partial x}\left(\frac{\partial \Omega_{2}}{\partial x}+\eta_{1} \frac{\partial^{2} \Omega_{1}}{\partial z \partial x}\right)\right\}=0 .
\end{aligned}
$$

The solution to order $\epsilon^{0}$ is the undisturbed state given by $\Omega_{0}=-U_{0} x, \phi_{0}=V_{0} z / b$.

For $\epsilon^{1}$ terms we designate

$$
\eta_{1}=\cos k x \text {. }
$$

This implies that $\epsilon$ has the dimension of length, being the amplitude of this component of the surface elevation. Condition (11) is then $\phi_{1}=\left(-V_{0} / b\right) \cos k x$ at $z=0$. Hence

$$
\phi_{1}=\frac{V_{0}}{b} \frac{\sinh k(z-b) \cos k x}{\sinh k b} .
$$

Also (7) gives $\partial \Omega_{1} / \partial z=k U_{0} \sin k x$ at $z=0$, and the solution for $\Omega_{1}$ becomes

$$
\Omega_{1}=U_{0} \frac{\sin k x \cosh k(z+a)}{\sinh k a} \text {. }
$$

The remaining condition (13) then shows that

$$
\rho k U_{0}^{2} \operatorname{coth} k a-T k^{2}-\rho g+\frac{V_{0}^{2} k}{4 \pi b^{2}} \operatorname{coth} k b=0,
$$

which gives us the value of $U_{0}$. A term of the form $U_{1} x$ could have been added to $\Omega_{1}$ in (17), but then (13) requires $U_{1}=0$.

We turn now to the conditions of order $\epsilon^{2}$. Using (15), (16), (17), we find that conditions (8) and (11) are, respectively,

$$
\begin{gathered}
\frac{\partial \Omega_{2}}{\partial z}+U_{0} \frac{d \eta_{2}}{d x}+k^{2} U_{0} \operatorname{coth} k a \sin 2 k x=0, \\
\phi_{2}+\frac{V_{0} \eta_{2}}{b}+\frac{V_{0} k}{2 b} \operatorname{coth} k b(1+\cos z k x)=0 .
\end{gathered}
$$

After some reduction (14) becomes

$$
\rho U_{0} \frac{\partial \Omega_{2}}{\partial x^{1}}+T \frac{d^{2} \eta_{2}}{d x^{2}}-\rho g \eta_{2}+\frac{V_{0}}{4 \pi b} \frac{\partial \phi_{2}}{\partial z}=l+m \cos 2 k x
$$

where

$$
l=\left(\frac{\rho k^{2} U_{0}^{2}}{4}-\frac{V_{0}^{2} k^{2}}{16 \pi b^{2}}\right)\left(\operatorname{coth}^{2} k a-1\right),
$$

and

$$
m=\left(\frac{\rho k^{2} U_{0}^{2}}{4}-\frac{V_{0}^{2} k^{2}}{16 \pi b^{2}}\right)\left(\operatorname{coth}^{2} k a-3\right)
$$

Since $\eta_{2}$ can have no mean part we write

$$
\eta_{2}=r \cos 2 k x+s \sin 2 k x \text {. }
$$


Also let

$$
\left.\begin{array}{l}
\phi_{2}=\alpha+\beta \cos 2 k x+\gamma \sin 2 k x \\
\Omega_{2}=\lambda x+\mu \cos 2 k n+\nu \sin 2 k x
\end{array}\right\} \text { at } z=0 .
$$

Solutions for $\phi_{2}$ and $\Omega_{2}$ are then

$$
\begin{gathered}
\phi_{2}=\alpha\left(1-\frac{z}{b}\right)-\frac{\sinh 2 k(z-b)}{\sinh 2 k b}\{\beta \cos 2 k x+\gamma \sin 2 k x\}, \\
\Omega_{2}=\lambda x+\frac{\cosh 2 k(z+a)}{\cosh 2 k a}(\mu \cos 2 k x+\gamma \sin 2 k x) .
\end{gathered}
$$

Eqs. (22), (23), (24) can then be used to satisfy (19), (20) and (21) at $z=0$, by ensuring that the coefficients of $\sin 2 k x, \cos 2 k x$, and the constant terms are zero in each. Eq. (19) gives

$$
\begin{aligned}
& 2 U_{0} r-2 \gamma \tanh 2 k a=U_{0} k \operatorname{coth} k a, \\
& 2 U_{0} s+2 \mu \tanh 2 k a=0 .
\end{aligned}
$$

From (20), we find

$$
\begin{gathered}
\alpha=-\frac{V_{0} k}{2 b} \operatorname{coth} k b, \\
\beta+\frac{V_{0} r}{b}+\frac{V_{0} k}{2 b} \operatorname{coth} k b=0, \\
\gamma+\frac{V_{0} s}{b}=0 .
\end{gathered}
$$

Eq. $(21)$ is satisfied when

$$
\begin{gathered}
\rho U_{0} \lambda-\frac{V_{0} \alpha}{4 \pi b^{2}}=l, \\
2 k \rho U_{0} \mu+4 k^{2} T s+\rho g s-\frac{2 k V_{0} \gamma}{4 \pi b} \operatorname{coth} 2 k b=0, \\
2 k \rho U_{0} \nu+4 k^{2} T r-\rho g r-2 k V_{0} \beta \frac{\operatorname{coth} 2 k b}{4 \pi b}=m .
\end{gathered}
$$

Eqs. (25)-(32) may now be solved for the unknown coefficients $\alpha, \beta, \gamma, \lambda, \mu, \nu, r$ and $s$. The coefficients of most interest are those which give rise to the mean changes to this order, namely $\alpha$ and $\lambda$. Eq. (23) shows that there is a second-order change in the mean electrostatic field given by

$$
\phi_{2}=-\frac{V_{0} k \operatorname{coth} k b}{2 b}\left(1-\frac{z}{b}\right) .
$$

Thus to the order $\epsilon^{2}$ the mean electric field is raised to

$$
\frac{V_{0}}{b}\left\{1+\frac{\epsilon^{2} k}{2 b} \operatorname{coth} k b\right\} \text {, }
$$


and this is accompanied by additional charge on the upper plate of amount

$$
\frac{V_{0} k \epsilon^{2}}{8 \pi b^{2}} \operatorname{coth} k b \text {. }
$$

The coefficient $\lambda$ measures the second-order change in the phase velocity of the wave. We have

$$
\rho U_{0} \lambda=l+\frac{V_{0}}{4 \pi b^{2}} \alpha=\left(\frac{\rho k^{2} U_{0}^{2}}{4}-\frac{V_{0}^{2} k^{2}}{16 \pi b^{2}}\right) \operatorname{cosech}^{2} k a-\frac{V_{0}^{2} k \operatorname{coth} k b}{8 \pi b^{3}} .
$$

This gives rise to an additional phase velocity $-\partial \Omega_{2} / \partial x=-\lambda$, so that to order $\epsilon^{2}$ the phase velocity of the wave is

$$
U=U_{0}-\frac{\epsilon^{2}}{\rho U_{0}}\left\{\left(\frac{\rho k^{2} U_{0}^{2}}{4}-\frac{V_{0}^{2} k^{2}}{16 \pi b^{2}}\right) \operatorname{cosech}^{2} k a-\frac{V_{0}^{2} k}{8 \pi b^{3}} \operatorname{coth} k b\right\} .
$$

When $V_{0}=0$ this reduces to the Stokes [2] result, and it is noticeable that the electrostatic effect is to increase this speed in the $\epsilon^{2}$ terms.

(b) Charge-maintained oscillations. The above analysis shows that when the potentials of the conducting surfaces are fixed a change in the mean charge density of order $\epsilon^{2}$ occurs. If the conductors are insulated this change cannot occur, and we might then expect that there will occur a change of order $\epsilon^{2}$ occurring the potential difference between the fluid and the plate instead.

When the conducting plate at $z=b$ is insulated, conditions (vii) and (viii) must now be replaced so that

$$
\phi_{1}=c_{1}, \quad \phi_{2}=c_{2}, \quad \phi_{3}=c_{3}, \cdots \quad \text { at } z=b,
$$

where $c_{1}, c_{2}, c_{3} \cdots$ are constants. This represents the condition that the potential at the plate is constant, but not necessarily unchanged in the wave motion. Further, $c_{1}, c_{2}, c_{3}$ $\cdots$ must be such that the total charge per wavelength is unchanged to all orders in $\epsilon$.

(x) Similarly, we have $\phi=$ constant at $z=\eta$, with the same condition of invariance on the total charge per wavelength.

We shall trace through the changes in the previous analysis when (ix) and (x) apply instead of (vii) and (viii), without repeating all the steps in full.

The boundary conditions on $\phi_{1}$ now become $\phi_{1}=c_{1}$ at $z=b$, and, instead of (10) $\phi_{1}+$ $\eta_{1}\left(\partial \phi_{0} / \partial z\right)=d_{1}$, say, at $z=0$, where $d_{1}$ is a constant. Evidently if $d_{1} \neq c_{1}$, a uniform electric field of order $\epsilon$ would be present, which would give a change in the mean surface charge density of order $\epsilon$, which is inadmissible. Hence $d_{1}=c_{1}$ and we may without loss of generality let $c_{1}=d_{1}=0$ on the basis that the fluid potential remains unchanged. Assuming $\eta_{1}$ again as in (15), this leaves the solution (16) for $\phi_{1}$ unchanged. Eq. (17) for $\Omega_{1}$ is also unchanged.

As boundary conditions for $\phi_{2}$ we now have $\phi_{2}=c_{2}$ at $z=b$, and $\phi_{2}+\eta_{2}\left(\partial \phi_{0} / \partial z\right)+$ $\eta_{1}\left(\partial \phi_{1} / \partial z\right)=d_{2}$ at $z=0$ instead of (11), where $d_{2}$ is another constant. Condition (19) remains unchanged, but for (20) we now have

$$
\phi_{2}+\frac{V_{0} \eta_{2}}{b}+\frac{V_{0} k}{2 b} \operatorname{coth} k b(1+\cos 2 k x)=d_{2} \quad \text { at } \quad z=0 .
$$

Eqs. (21) and (22) are unaltered, so now 


$$
\phi_{2}=d_{2}-\frac{V_{0}}{b}(r \cos 2 k x+s \sin 2 k x)-\frac{V_{0} k}{2 b} \operatorname{coth} k b(1+\cos 2 k x) \quad \text { at } \quad z=0 .
$$

No change in the mean value of $\phi_{2}$ can occur between $z=0$ and $z=b$ in this case, since there can be no change in mean charge density. Hence

$$
d_{2}-\frac{V_{0} k}{2 b} \operatorname{coth} k b=c_{2} \text {. }
$$

With the fluid at earthed potential, we take $d_{2}=0$ and $c_{2}=\left(-V_{0} k / 2 b\right) \operatorname{coth} k b$. Thus

$$
\phi_{2}=-\frac{V_{0} k}{2 b} \operatorname{coth} k b-\frac{\sinh 2 k(z-b)}{\sinh 2 k b}\{\beta \cos 2 k x+\gamma \sin 2 k x\},
$$

which shows a mean loss in potential difference $\left(\epsilon^{2} V_{0} k / 2 b\right) \operatorname{coth} k b$, to the order $\epsilon^{2}$. The solution for $\Omega_{2}$ given by (24) is unaltered, and (22), (24) and (35) must now be used to satisfy the conditions (19), (20) and (21) at $z=0$. We find that Eqs. (25), (26), (28), (29), (31) and (32) are unaltered. But (30) is changed because $\partial \phi_{2} / \partial z$ now has no constant component, so that now

$$
\rho U_{0} \lambda=l=\frac{1}{\sinh ^{2} k a}\left(\frac{\rho k^{2} U_{0}^{2}}{4}-\frac{V_{0}^{2} k^{2}}{16 \pi b^{2}}\right) .
$$

The second-order contribution to the phase velocity is therefore lower, and we have

$$
U=U_{0}-\frac{\epsilon^{2}}{\rho U_{0}}\left(\frac{\rho k^{2} U_{0}^{2}}{4}-\frac{V_{0}^{2} k^{2}}{16 \pi b^{2}}\right) \operatorname{cosech}^{2} k a, \text { to order } \epsilon^{2} .
$$

We note that, otherwise, the solution for $\beta, \gamma, \mu, \nu, r, s$ remains the same as in case (a), so that, for example, there is no distinction at this order between the surface elevation or the motion of the fluid in the wave, as seen relative to the rest frame in each case.

3. Unstable standing waves. When the phase velocity $U_{0}$ is imaginary, wave propagation without change of form becomes impossible, and progressive waves with phase of the form $\exp i(k x \pm \omega t)$ change to the form $\exp (i k x \pm \sigma t)$ where $\sigma$ is real. The most natural form of wave function to study in this case is of the form $f(x) \exp (c t)$, where again $f(x)$ is a periodic function with wavelength $2 \pi / k$. The approach to this problem must change because there will not exist a frame of reference in which the motion is steady. There is then no advantage in changing the frame of reference from the natural one in which the fluid is at rest in the absence of the wave. However, there is the complication in this case that the wave motion is now unsteady. As in Sec. 2, we shall consider the growth of the wave in the different electrostatic conditions, but without repeating steps which are the same as in Sec. 2.

We may again write expansions for $\phi, \eta$ as (1) and (2), with $\phi_{0}=V_{0} z / b$, but (3) we now write as

$$
\Omega=\epsilon \Omega_{1}+\epsilon^{2} \Omega_{2}+\epsilon^{3} \Omega_{3}+\cdots .
$$

The terms in $\Omega$ are now unsteady.

Conditions (i), (ii), (iii), (iv) and (vi) remain, but (v) now becomes

(xi) $\left(\frac{\partial \Omega}{\partial n}\right)_{z=y}=$ normal velocity of the surface. 
As in Sec. 2 we make a distinction between the electrostatic conditions under subsections (a) and (b).

(a) Waves at fixed potentials. Conditions (vii) and (viii) will apply in this case. Condition (xi) may be written

$$
\left(\frac{\partial \Omega}{\partial z} \cos \psi-\frac{\partial \Omega}{\partial x} \sin \psi\right)=\frac{\partial \eta}{\partial t} \cos \psi,
$$

or

$$
\left(\frac{\partial \Omega}{\partial z}-\frac{\partial \zeta}{\partial x} \frac{\partial \Omega}{\partial x}\right)=\frac{\partial \eta}{\partial t} .
$$

Now we suppose that the wave growth occurs on a time scale $c$ where

$$
c=c_{0}+\epsilon c_{1}+\epsilon^{2} c_{2}+\cdots,
$$

and write $t=c \tau$ where $\partial / \partial \tau \sim 1$. Then

$$
\left(c_{0}+\epsilon c_{1}+\epsilon^{2} c_{2}+\cdots\right)\left(\frac{\partial \Omega}{\partial z}-\frac{\partial \eta}{\partial x} \frac{\partial \Omega}{\partial x}\right)=\frac{\partial \eta}{\partial t} \quad \text { at } \quad z=\eta .
$$

To order $\epsilon^{2}$ this becomes

$$
\begin{array}{ll}
\epsilon^{1}: & c_{0}\left(\frac{\partial \Omega_{1}}{\partial z}\right)=\frac{\partial \eta_{1}}{\partial \tau} \\
\epsilon^{2}: & c_{1} \frac{\partial \Omega_{1}}{\partial z}+c_{0}\left(\frac{\partial \Omega_{2}}{\partial z}+\eta_{1} \frac{\partial^{2} \Omega_{1}}{\partial z_{2}}-\frac{\partial \Omega_{1}}{\partial x} \frac{\partial \eta_{1}}{\partial x}\right)=\frac{\partial \eta_{2}}{\partial \tau} .
\end{array}
$$

Conditions (39), (40) replace (7) and (8). The equations (9), (10) and (11) for condition (viii) remain unchanged. But the stress condition (vi) now takes a different form because there is a contribution to the pressure from the unsteadiness of the fluid motion. We have

$$
p=f(t)-\frac{1}{2} \rho(\operatorname{grad} \Omega)^{2}-\rho(\partial \Omega / \partial t) .
$$

When this is put in the surface stress condition, and when we allow for the expansion of $c$ in powers of $\epsilon_{1}$, we find to order $\epsilon^{2}$ :

$$
\begin{gathered}
\epsilon^{0}: \quad \frac{1}{8 \pi}\left(\frac{\partial \phi_{0}}{\partial z}\right)^{2}=\text { constant } \\
\epsilon^{1:} \quad c_{0}\left(T \frac{\partial^{2} \eta_{1}}{\partial x^{2}}-\rho g \eta_{1}+\frac{1}{4 \pi} \frac{\partial \phi_{0}}{\partial z} \frac{\partial \phi_{1}}{\partial z}\right)-\rho \frac{\partial \Omega_{1}}{\partial \tau}=0 \\
\epsilon^{2}: \quad c_{0}\left\{T \frac{\partial^{2} \eta_{2}}{\partial x^{2}}-\rho g \eta_{2}-\frac{\rho}{2}\left\{\left(\frac{\partial \Omega_{1}}{\partial x}\right)^{2}+\left(\frac{\partial \Omega_{1}}{\partial z}\right)^{2}\right\}\right. \\
\left.+\frac{1}{8 \pi}\left\{\left(\frac{\partial \phi_{1}}{\partial z}\right)^{2}+2 \frac{\partial \phi_{0}}{\partial z}\left(\frac{\partial \phi_{2}}{\partial z}+\eta_{1} \frac{\partial^{2} \phi_{1}}{\partial z^{2}}-\frac{\partial \eta_{1}}{\partial x} \frac{\partial \phi_{1}}{\partial x}\right)-\left(\frac{\partial \phi_{0}}{\partial z}\right)^{2}\left(\frac{\partial \eta_{1}}{\partial x}\right)^{2}\right\}\right\} \\
+c_{1}\left\{T \frac{\partial \eta_{1}}{\partial x^{2}}-\rho g \eta_{1}+\frac{1}{4 \pi} \frac{\partial \phi_{0}}{\partial z} \frac{\partial \phi_{1}}{\partial z}\right\}-\rho \frac{\partial \Omega_{2}}{\partial \tau}-\rho \eta_{1} \frac{\partial^{2} \Omega_{1}}{\partial z}=0 .
\end{gathered}
$$

Eqs. (41), (42) and (43) replace (12), (13) and (14). 
For the first-order solution let

$$
\eta_{1}=g(\tau) \cos k x .
$$

It follows from (10) that $\phi_{1}=\left(-V_{0} / b\right) g(\tau) \cos k x$ at $z=0$. Thus

$$
\phi_{1}=\frac{V_{0}}{b} g(\tau) \frac{\sinh k(z-b)}{\sinh k b} \cos k x .
$$

Also following from (39) and (44) we find

$$
\Omega_{1}=\frac{g^{\prime}(\tau) \cosh k(z+a) \cos k x}{k c_{0} \sinh k a},
$$

where $g^{\prime}(\tau)=d g / d \tau, g^{\prime \prime}(\tau)=d^{2} g / d \tau^{2}$, etc. Eq. (42) gives

$$
g^{\prime \prime}(\tau)=k c_{0}^{2} \tanh k a\left[\frac{V_{0}^{2} k}{4 \pi b^{2}} \operatorname{coth} k b-T k^{2}-\rho g\right] g(\tau) .
$$

The appropriate form of solution is to let $c_{0}$ be determined by the equation

$$
k c_{0}{ }^{2} \tanh k a\left[\frac{V_{0}^{2} k}{4 \pi b^{2}} \operatorname{coth} k b-T k^{2}-\rho g\right]=1,
$$

so that $g^{\prime \prime}(\tau)=g(\tau)$. The condition for instability in linear form, is that ${c_{0}}^{2}>0$; that is,

$$
\frac{V_{0}^{2} k}{4 \pi b^{2}} \operatorname{coth} k b>T k^{2}+\rho g \text {. }
$$

For the $\epsilon^{2}$ terms we have from (11)

$$
\phi_{2}+\frac{V_{0}}{b} \eta_{2}+\frac{k V_{0}}{2 b} \operatorname{coth} k b g^{2}(\tau)(1+\cos 2 k x)=0, \quad(z=0) .
$$

We note that $\eta_{2}$ can be of the form

$$
\begin{array}{l|l}
\sin & 2 k x \\
\cos &
\end{array}
$$

with no mean part. Hence the mean part of the last term of (48) must be matched by a mean part of $\phi_{2}$ at $z=0$. Now (40) becomes, after substitution,

$$
\frac{\partial \eta_{2}}{\partial \tau}-c_{0} \frac{\partial \Omega_{2}}{\partial z}-k g g^{\prime} \operatorname{coth} k a \cos 2 k x-\frac{c_{1}}{c_{0}} \cos k x g^{\prime}(\tau)=0 .
$$

Since $\Omega_{2}$, like $\eta_{2}$, can contain only terms in $x$ of the form

$$
\sin \mid 2 k x
$$

the $\cos k x$ term in (49) is unbalanced, and therefore $c_{1}=0$, so that

$$
\frac{\partial \eta_{2}}{\partial \tau}-c_{0} \frac{\partial \Omega_{2}}{\partial z}=k g g^{\prime} \operatorname{coth} k a \cos 2 k x
$$

The last condition of order $\epsilon^{2}$ is (43) which becomes, after substitution,

$$
\frac{\partial^{2} \eta_{2}}{\partial x^{2}}-\rho g \eta_{2}-\frac{\rho}{c_{0}} \frac{\partial \Omega_{2}}{\partial \tau}+\frac{V_{0}}{4 \pi b}\left(\frac{\partial \phi_{2}}{\partial z}\right)=\frac{\rho\left(g^{\prime}\right)^{2}}{4 c_{0}^{2}}\left\{\left(\operatorname{coth}^{2} k a+1\right)+\left(1-\operatorname{coth}^{2} k a\right) \cos 2 k x\right\}
$$


$-\frac{V_{0}^{2} k^{2} g^{2}}{16 \pi b^{2}}\left[\left(\operatorname{coth}^{2} k a-1\right)+\left(\operatorname{coth}^{2} k b-3\right) \cos 2 k x\right]+\frac{\rho g g^{\prime \prime}}{2 c_{0}{ }^{2}}(1+\cos 2 k x), \quad$ at $\quad z=0$.

Eq. (51) shows that $\Omega_{2}$ has a component which is a function of $\tau$ only which must be used to match the terms independent of $x$ on the right-hand side of (51). This does not affect the velocity distribution, but does represent a change in the mean pressure to the second order.

We shall confine our discussion at this stage to the mean components, which are of most interest. Clearly from (48) a second-order mean electric field arises from the requirements that

$$
\begin{array}{ll}
\phi_{2}=-\frac{k V_{0}}{2 b} g^{2}(\tau) \operatorname{coth} k b \text { at } z=0, \\
\phi_{2}=0 & \text { at } z=b .
\end{array}
$$

This solution is therefore

$$
\phi_{2}=\frac{k V_{0}}{2 b} g^{2}(\tau) \operatorname{coth} k b\left(\frac{z}{b}-1\right),
$$

and represents an increase in the mean field to

$$
\frac{V_{0}}{b}\left\{1+\frac{\epsilon^{2} k}{2 b} g^{2}(\tau) \operatorname{coth} k b\right\} \text {. }
$$

From (51) we find that the mean part of $\partial \Omega_{2} / \partial \tau$ is given by

$$
-\rho \frac{\partial \Omega_{2}}{\partial \tau}=\frac{\rho\left(g^{\prime}\right)^{2}}{4 c_{0}}\left(\operatorname{coth}^{2} k a+1\right)+\frac{\rho g g^{\prime \prime}}{2 c_{0}}-\frac{V_{0}^{2} k^{2} g^{2}(\tau) c_{0}}{16 \pi b^{2}}\left\{\operatorname{coth}^{2} k b+\frac{2 \operatorname{coth} k b}{k b}-1\right\} \text {. }
$$

Thus the right-hand side of (52) represents the increase in mean pressure to the second order.

For the parts of the second-order solution periodic in $x$ we again write $\eta_{2}, \phi_{2}, \Omega_{2}$ in terms of

$$
\begin{array}{c|c}
\sin \\
\cos
\end{array} 2 k x \text { with the coefficients } r, s, \beta, \gamma, \mu, \nu
$$

as used in Sec. 2. But here these coefficients are functions of $\tau$. The periodic parts of $\phi_{2}$ and $\Omega_{2}$ follow as in (23) and (24). The equations for the coefficients are now

$$
\begin{gathered}
\beta+\frac{V_{0}}{b} r+\frac{k V_{0}}{2 b} \operatorname{coth} k b[g(\tau)]^{2}=0, \\
\gamma+\frac{V_{0}}{b} s=0, \\
\frac{d r}{d \tau}-2 k c_{0} \mu \tanh 2 k a-k h g g^{\prime} \operatorname{coth} k a=0,
\end{gathered}
$$




$$
\frac{d s}{d \tau}-2 k c_{0} \nu \tanh 2 k a=0
$$

$$
\begin{aligned}
-4 k^{2} T r-\rho g r & -\frac{\rho d \mu}{c_{0} d \tau}-\frac{V_{0}}{4 \pi b} \cdot 2 k \beta \operatorname{coth} 2 k b \\
= & \frac{\rho\left(g^{\prime}\right)^{2}}{4 c_{0}^{2}}\left(1-\operatorname{coth}^{2} k a\right)+\frac{\rho g g^{\prime \prime}}{2 c_{0}^{2}}-\frac{V_{0}^{2} k^{2} g^{2}}{16 \pi b^{2}} \quad\left(\operatorname{coth}^{2} k b-3\right), \\
& -4 k^{2} T s-\rho g s-\frac{\rho}{c_{0}} \frac{d \nu}{d \tau}-\frac{V_{0}}{4 \pi b} 2 k \gamma \operatorname{coth} 2 k b=0 .
\end{aligned}
$$

(b) Charge-maintained oscillations. The changes needed to apply the alternative boundary conditions on $\phi$ follow much as in Sec. 2. It is easily seen that (45) for $\phi$ still holds, with the proviso that the fluid is at earthed potential. Also, Eq. (46) for $\Omega_{1}$ and the equation for $g(\tau)$ are unchanged. With the same significance for $c_{2}$ and $d_{2}$ as in Sec. 2, we now have

$$
d_{2}-\frac{k V_{0}}{2 b} g^{2}(\tau) \operatorname{coth} k b=c_{2}
$$

or, with $d_{2}=0$,

$$
c_{2}=-\frac{k V_{0}}{2 b} \operatorname{coth} k b g^{2}(\tau)
$$

Thus the mean loss of potential is $\left(\epsilon^{2} V_{0} / 2 b\right)[g(\tau)]^{2}$ coth $k b$ in this case. Eqs. (48) and (49) remain. So also does (51), though now, since $\partial \phi_{2} / \partial z$ has no mean part, the equation for the mean pressure change is now

$$
-\frac{\rho \partial \Omega_{2}}{\partial t}=\frac{\rho\left(g^{\prime}\right)^{2}}{4 c_{0}}\left(\operatorname{coth}^{2} k a+1\right)+\frac{\rho g g^{\prime \prime}}{2 c_{0}}-\frac{V_{0}{ }^{2} k^{2} g^{2} c_{0}}{16 \pi b^{2}}\left\{\operatorname{coth}^{2} k b-1\right\} .
$$

The increase in mean pressure in (59) as compared with (52) is due directly to the absence of electrostatic stress at the free surface arising from the mean part of $\phi_{2}$. Eqs. (53)-(58) for the periodic coefficients remain unaltered, and we see that the form of the free-surface is again the same to order $\epsilon^{2}$ in both cases $(a)$ and $(b)$. As a final remark we note that $g(\tau)$ has been left unspecified. Its particular form is determined by the initial conditions.

\section{REFERENCES}

[1] D. H. Michael, Note on electrohydrodynamic stability, Quart. Appl. Math. 28, 139-143 (1970)

[2] G. G. Stokes, On the theory of oscillatory waves, in Mathematical and physical papers, Cambridge University Press, 1880, vol. 1, p. 197 